BULLETIN OF THE

AMERICAN MATHEMATICAL SOCIETY

Volume 79, Number 6, November 1973

\title{
SOME POLYNOMIAL ALGEBRAS OVER THE STEENROD ALGEBRA $A_{p}$
}

\author{
BY CLARENCE WILKERSON
}

Communicated by Morton Curtis, March 5, 1973

The problem of classifying the possible cohomology algebras of topological spaces has usually been attacked negatively by introducing cohomology operations and requiring these to be compatible with the structure of the candidate for a cohomology algebra. Even in the special case that the $\boldsymbol{Z} / p$ cohomology is a polynomial algebra, the information obtained in this way is limited. For example, Steenrod [3] suggested a method of verifying that a given $Z / p$ polynomial algebra is a module over the Steenrod algebra $A_{p}$ and carried out the computations for

$$
\left\{(Z / p)\left[x_{4}, y_{4}+{ }_{2(p-1)}\right], P_{p}^{1} x=y\right\} .
$$

Such an approach has the drawbacks of being tedious to apply and also not producing a space with the given cohomology algebra. In this note, some ideas from Lie group theory and a generalization of a construction of Sullivan [4] are used to expand the rather small list of known polynomial cohomology algebras. In particular, the example treated by Steenrod is realized as the $Z / p$ cohomology of a space. In a later paper [5] the analogy of this construction with some properties of the Lie groups will be pursued and an application to the construction of some new mod $p$ loop spaces given.

Notation. $\boldsymbol{Z} / p=\{$ integers $\bmod p\}$,

$\hat{\mathbf{Z}}_{p}=\{p$-adic integers $\}$,

$\boldsymbol{Q}_{p}=\{p$-adic numbers $\}$,

$K\left(\hat{\boldsymbol{Z}}_{p}, 2\right)=$ Eilenberg-Mac Lane space of type $\left(\hat{\boldsymbol{Z}}_{p}, 2\right)$.

LeMma 1.1. $H^{*}\left(K\left(\hat{\boldsymbol{Z}}_{p}, 2\right), \boldsymbol{Z} / p\right)=(\boldsymbol{Z} / p)[x], \operatorname{dim} x=2$.

Definition 1.2 [2]. Let $W$ be a finite subgroup of $G L\left(Q_{p}, n\right) . W$ is generated by reflections in $Q_{p}^{n}$ if $W$ is generated by elements which have a hyperplane pointwise invariant. These elements thus have $n-1$ eigenvalues of +1 and the remaining eigenvalue an $r$ th root of unity ( $r$ must of course divide $p-1)$. Also, since $W$ is finite, we may consider it to be in $G L\left(\hat{\boldsymbol{Z}}_{p}, n\right)$.

Construction 1.3. Let $W$ be generated by reflections in $\boldsymbol{Q}_{p}^{n}$. By choosing an action of $W$ on $\pi_{2}\left(K\left(\oplus_{n} \hat{\boldsymbol{z}}_{p}, 2\right)\right), W$ may be given an action on

AMS (MOS) subject classifications (1970). Primary 55G10, 57F25.

Copyright (C) American Mathematical Society 1974 
$K\left(\oplus_{n} \hat{\boldsymbol{Z}}_{p}, 2\right)$ as a group of cellular homeomorphisms. The orbit space $X=K\left(\oplus_{n} \hat{Z}_{p}, 2\right) / W$ can then be formed.

It remains to calculate $H^{*}(X, \boldsymbol{Z} / p)$. This follows, however, from Chevalley [1].

THEOREM 2.1. If $W$ is generated by reflections, $p \nmid|W|$, and $X=$ $K\left(\oplus_{n} \hat{Z}_{p}, 2\right) / W$, then $H^{*}(X, Z / p)$ is a polynomial algebra on $n$ generators.

REMARK. The hypothesis that $p \nmid|W|$ is too strong, as examples with $W=S_{n}$ (the symmetric group) show, but the author does not know a more general sufficient hypothesis.

To apply Theorem 2.1, one needs a good supply of finite reflection groups on $\boldsymbol{Q}_{p}^{n}$. The Weyl groups of the Lie groups qualify, since these are precisely the finite reflection groups on $Q^{n}$, but these produce no new cohomology algebras. It is not hard to see that a $p$-adic representation of a finite group is equivalent to one over a finite extension of the rationals contained in the $p$-adics, and thus may be regarded as a complex representation. The paper [2] of Shephard and Todd classifies the finite reflection groups over the complexes, and thus, in a sense, classifies all finite reflection groups over $\boldsymbol{Q}_{p}^{n}$. One need only verify if a particular group from [2] has a $p$-adic representation. This can involve surprisingly difficult number theoretic calculations. For example, the fact that the group $W$ presented as $\left\{A^{2}=B^{2}=(A B)^{p+1}=\mathrm{Id}\right\}$ has a representation as a $p$-adic reflection group reduces to the following lemma.

LEMMA 2.2 [6]. Let $K$ be an extension field of $\boldsymbol{Q}_{p}$ containing $\theta$, a $(p+1)$ st root of unity. Then $\theta+\theta^{-1} \in \widehat{\mathbf{Z}}_{p}$.

The immediate consequence is:

COROLlaRY 2.3. There exists a space $X$ with

$$
H^{*}(X, Z / p)=(Z / p)\left[x_{4}, y_{4}+{ }_{2(p-1)}\right] \text { and } P_{p}^{1}(x)=y .
$$

Proof. Take $A=\left(\begin{array}{ll}0 & 1 \\ 1 & 0\end{array}\right)$ and $B=\left(\begin{array}{cc}-1 & 0 \\ \theta+\theta^{-1} & 1\end{array}\right)$. The dimensions of the invariants are given in [2], and a brief calculation shows that $P_{p}^{1}(x)=y$.

In [5], a more complete list of these $\mathbf{Z} / p$ polynomial algebras will be compiled.

\section{REFERENCES}

1. C. Chevalley, Invariants of finite groups generated by reflections, Amer. J. Math. 77 (1955), 778-782. MR 17, 345, 1436.

2. G. C. Shephard and J. A. Todd, Finite unitary reflection groups, Canad. J. Math. 6 (1954), 274-304. MR 15, 600.

3. N. E. Steenrod, Polynomial algebras over the algebra of cohomology operations, $H$ spaces (Actes Réunion Neuchâtel, 1970), Lecture Notes in Math., vol. 196, Springer, Berlin, 1971, pp. 85-99. MR 44 \# 3316. 
4. D. Sullivan, Localization, periodicity and Galois symmetry, M.I.T., Cambridge, Mass., 1971.

5. C. Wilkerson, Maximal tori and Weyl groups in mod p loop spaces (in preparation).

6. D. Zagier, Private communication.

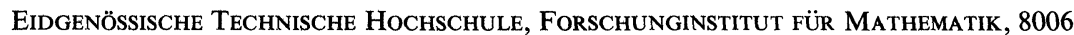
ZURICH, SWITZERLAND

Current address: Department of Mathematics, Carleton University, Ottawa K1S 5B6 Ontario, Canada 